\title{
Myeloid-derived suppressor cells in animal transplantation models
}

\begin{abstract}
Myeloid-derived suppressor cells" have been introduced to scientific world for more than 10years. They are a heterogeneous population of cells that gain immune suppression function in a pathologic state, such as tumor, chronic infection, obesity et al. The group of cells suppress the immune system through a variety of ways, such as Arg-1, HO-1, NOS, ROS and NOX-2. MDSCs can also work together with other immunosuppressive cell, such as Treg and Breg to induce immune tolerance. In kidney, heart, skin, bone marrow, islet and other organ transplant of animal models, MDSCs can reduce graft rejection in different mechanism. This article reviews the progress of MDSCs in the field of animal transplantation and discusses its potential clinical application
\end{abstract}

Volume 3 Issue I - 2018

\author{
Bo Wan,' Qing Li, ${ }^{2}$ Xiao Fan $\mathrm{Li}^{3}$ \\ 'The First Affiliated Hospital of Fujian Medical University, China \\ ${ }^{2}$ Department of Obstetrics and Gynecology, Fujian Medical \\ University Provincial Clinical College, China \\ ${ }^{3}$ Department of Hematology, Fujian Medical University Union \\ Hospital, China \\ \#-All the authors contributed equally to this article.
}

Correspondence: Xiao Fan Li, Fujian Institute of Hematology, Fujian Provincial Key Laboratory on Hematology, Department of Hematology, Fujian Medical University Union Hospital, Fuzhou, Fujian, PR: 350000, China, Email morningshiplee@sina.com

Received: January 25, 2018 | Published: February |4, 2018

\section{Introduction}

Myeloid-derived suppressor cells (MDSCs) are a heterogeneous population of immunosuppressive cells of the myeloid lineage, consisting of progenitor cells of macrophages, granulocytes, dendritic cells (DC) and immature myeloid cells (IMC). ${ }^{1-3}$ There are many researches on tumor MDSCs and negative effect on immune system. ${ }^{4,5}$ Because of the negative regulation of immune response through different ways, MDSCs related researches are performed by transplant immunologists. ${ }^{6,7}$ Many research find that MDSCs can prevent graft rejection reaction, and induce immune tolerance in kidney, heart, skin, bone marrow, islet transplantation. ${ }^{8,9}$ Herein, we review the immunosuppressive role in animal transplantation models, as well as its potential clinical application prospect.

\section{Characteristics and roles of MDSCs in animal transplantation}

MDSCs were first found in cancer and determined to suppress immune responses to tumors. There are three important characteristics: myeloid origin; immature state; T cell suppression. They are a group of immature, heterogeneous cell populations that include myeloid precursor cells, immature granulocytes, macrophages, dendritic cells, etc. Hematopoietic stem cells differentiate into common myeloid precursors, and then immature myeloid cells. In normal conditions, immature myeloid cells differentiate into neutrophils, macrophages, and DC. After transplantation, especially in chronic infection/inflammation, ${ }^{10}$ immature myeloid cells differentiate into MDSCs and gain immunosuppressive functions. ${ }^{11}$ These processes are not completely understood yet. It seems to be associated with cytokine (GM-CSF, IFN, IL-2, TGF-beta), interferon regulatory transcription factor,CXCL1/2, PGE2 Waight JD et al. ${ }^{12}$ In mice, MDSCs phenotypes were defined as CD11b Gr1 cell subset. Within mouse MDSCs, There are two primary sub-populations: monocytic MDSCs (M-MDSCs) and polymer pronuclear MDSCs (PMNMDSC). The M-MDSCs are CD11b+ly6G-Ly6Chigh, are bona fide phagocytes and are morphologically similar to monocytes, while exerting localized suppressive activity. ${ }^{13}$ The PMN-MDSCs are
CD11b+Ly6G+Lyr6Clow. ${ }^{14}$ Mouse PMN-MDSCs do not express CD49d, whereas M-MDSCs do. Also, cell surface expression of Ly6C and CD $11 \mathrm{~b}$ are slightly lower for PMN-MDSC than non-MDSC PMN, whereas CD115 and CD224 are higher for PMN-MDSC than non-MDSC PMN in mice. ${ }^{15}$

\section{The suppression mechanism of MDSCs in transplantation immunology}

MDSCs can suppress the $\mathrm{T}$ cell immunoresponse, which is important for the definition. Both cell surface contacts and soluble factors are important for suppress function. However, the mechanism may vary in different MDSCs in different transplantation types, as MDSC effector function is depending on the stimulus and environments. Many Researches has demonstrated that the action of arginase-1(Arg-1) and arginine, a nonessential amino acid, is important. Arginine plays an important role in the treatment via clinical immunotherapy. Arginine can increase T lymphocyte mediated cellular immunity, including delayed hypersensitivity. It also enhances the activity of monocyte and macrophage. It is reported that MDSCs have high expression of L - arginine metabolism enzyme Arg-1. Arg-1 catalyzes the transformation of Arginine into urea and L-ornithine. In mouse skin transplants, the migration of MDSC to the site of the skin graft can significantly prolong the survival of the skin graft. MDSCs upregulate the expression of Arg-1. Arg-1 acted on allo-antigen activated T lymphocytes, decreasd IL2 expression, decreasd cytotoxic $\mathrm{T}$ lymphocyte development and inhibited the T cell proliferation. ${ }^{16}$ In the MHC mismatch mouse bone marrow transplantation model, MDSCs inhibit T cell allogenic reaction and graft-versus-host disease by consuming Arginine. Arg1 deficient MDSCs showed impaired ability to the inhibition effect of GVHD. MDSC reduces the function of T cells by reducing the production of IFN, thus reducing the damage caused by delayed hypersensitivity, and inflammation to the graft. ${ }^{17}$ It is also interesting that extracorporeal photopheresis can increase T-cell-dampening PMN-MDSCs in GvHD patients. ${ }^{18}$

Heme oxygenase $(\mathrm{HO})$ can catalyze the oxidation of heme. It is the rate limiting enzyme of heme catabolism. HO-1 is recognized as 
immune modulatory characteristics and anti-inflammatory effects. HO-1 is expressed on monocytes, macrophages and bone marrow cells and can be up regulated in fever, shock or stress. HO-1 gene transfection can reduce ischemia-reperfusion injury, which effectively inhibits cardiac rejection in heart transplantation. In a mouse skin transplant, MDSCs produce a large number of IL 10 and HO-1, which is related to the activity of MDSCs. HO-1 can prevent dendritic cells from maturation and promote IL 10 secretion. Meanwhile, IL10 can also induce HO-1 expression. The use of the $\mathrm{HO}-1$ specific inhibitor SnPP can block the inhibitory effect of MDSC on T cells and reduce IL10 production by MDSC. Mixed lymphocyte reactions and polyclonal stimulation tests showed that MDSCs inhibited the production of Thl and Th 2 cytokines, and further prolonged the survival time of the skin graft. ${ }^{19}$

Nitric oxide synthase (NOS) is the key enzyme and rate-limiting enzyme in the synthesis of nitric oxide (NO), and its biological activity directly determines the amount of NO synthesized. Inducible nitric oxide synthase (iNOS) mainly exits in immune cells, macrophages. In animal kidney transplant, MDSCs accumulate in peripheral blood and tolerable kidney grafts. MDSCs induce effector T cell apoptosis through the over expression of iNOS and NO. iNOS acts by reducing the phosphorylation of tyrosine residues on JAK3 and STAT5 and induce reversible $\mathrm{T}$ cell energy. Inhibition of iNOS expression can damage the established tolerance state, leading to kidney graft rejection. ${ }^{20}$ In vitro experiments showed MDSCs significantly inhibited effectors $\mathrm{T}$ cell proliferation and induced apoptosis in a contact-dependent manner. Although adoptive transfer of MDSCs failed to induce renal allograft immune tolerance, the maintenance of transplant tolerance established by anti-CD28 antibody was dependent on iNOS activity. ${ }^{21}$ There is no rejection in mouse liver transplantation. However, hepatocyte transplant could be rejected, indicating that nonparenchymal liver cells have immune modulatory properties. hepatic stellate Cells are among those that have strong immunosuppressive properties..$^{22} \mathrm{MDSC}$ can be induced in vitro by adding mouse hepatic stellate cells into mouse bone marrow cells. Those MDSCs induced by hepatic stellate cells are highly expressed in iNOS, which can significantly inhibit the proliferation of T cells. iNOS increases the metabolism of Arginine and the decrease and consumption of arginine inhibits the function of T cells. The mechanism of Arginine depletion inhibiting $\mathrm{T}$ cell function may also be the inhibition of T cell Cyclin D3 and cyclin dependent kinase 4 (Cdk4) expressions, which eventually leads to cell cycle arrest in $\mathrm{T}$ cells, which inhibits the proliferation of T cells. ${ }^{23}$ The co-transplantation of MDSCs and islets of iNOStransgenic mice can also prolong the survival of the grafts compared with the islet transplantation alone, indicating that MDSCs has other mechanisms besides iNOS to regulate the immune response..$^{22}$ The other mechanism includes as follows. MDSCs deplete local levels of cysteine through sequestration. MDSCs also deplete reactive oxygen species (ROS) by NADPH oxidase-2 (Nox2), which all lead to suppression of T cells. MDSCs produce peroxynitrite (PNT), down regulate L-selection, and inhibit lymphocyte trafficking. ${ }^{13}$

\section{MDSCs and T cells}

A separate lineage of CD4+ $\mathrm{T}$ cells have been identified as regulatory $\mathrm{T}$ cells that can suppress activation and expansion of effector immune cells. Regulatory $\mathrm{T}$ cells constitutively express high levels of CD25, and CTLA4, and a transcription factor, Foxp3, which control regulatory $\mathrm{T}$ cell (Treg) development and suppressive function. Mutation of Foxp3 in scurfy mice leads to severe lethal autoimmune inflammation. Treg cells are demonstrated to be a dominant suppressor cell that is critical for peripheral tolerance. ${ }^{24}$ In animal kidney transplantation, blood-derived MDSCs were found to significantly down-regulate the chemokine CCL5 of immunetolerant receptors. MDSCs caused a CCL5 concentration gradient that tolerated animal grafts, resulting in the recruitment of Tregs in the graft to induce immune tolerance. ${ }^{25}$ In a mouse heart transplant model, mononuclear MDSCs migrate from the bone marrow to the transplanted organ, preventing the initiation immune responses and participating in the differentiation and maturation of Tregs. ${ }^{26}$ In animal islet transplantation, immunosuppressive medicine in combination with MDSCs can effectively induce large numbers of regulatory $T$ cells. In this model, MDSCs through the PDL1 signaling pathway could be directly induced by regulatory T cells. ${ }^{27}$ In vivo tests showed that the accumulation of MDSCs after kidney transplantation and Treg increased linearly. In vitro experiments show that MDSCs can amplify Treg. ${ }^{28}$ It is interesting that MDSCs also induce expansion of regulatory B cells and ameliorate autoimmunity in a murine model of SLE. 29

\section{Adoptive transfer of MDSCs}

In view of the immunosuppressive characteristics of MDSCs, it is necessary to induce, amplify and activate MDSCs in transplantation immunology. Adoptive transfer of MDSCs produced in vitro was used to control alloantigen specific reactions or induce immune tolerance. MDSCs can prolong graft survival in corneal transplantation models. ${ }^{30}$ The adoptive transfer of MDSCs can inhibit donor $\mathrm{T}$ cell infiltration of corneal grafts, which led to a reduction in the histopathological changes in the corneal allograft. ${ }^{31}$ Similar results were also found in mouse islet transplantation. ${ }^{32}$ Further, administration of MDSCs increased the number of Tregs within the islet graft..$^{33}$ In animal skin transplantation, the suppressive effects of the adoptively transferred MDSCs were dose-dependent. ${ }^{34,35}$ Compared to 1 million cells group, graft survival was prolonged by $50 \%$ when the dose of MDSCs was increased to 3 million cells. ${ }^{36}$

\section{Discussion}

MDSCs have potential clinical application benefits to transplanted patients. With ongoing research, we can now more clearly define the MDSC subset by cell surface markers and we are now clearer on the differentiation and migration of MDSCs developed after transplantation. However, there are many issues unanswered, such as whether the MDSCs after transplantation are antigen specific. Also, the translational expansion method of MDSCs ex vivo and the yield of MDSCs to infuse is different clinical transplantation situations are not known yet. It is of clinical significance to augment naturally occurring recipient MDSCs after transplantation, such that immune suppression dosing can be reduced or eliminated altogether. Up till now, majority of the MDSCs studies are done in small animals, monkey trials and human studies are needed for further clinical usage.

\section{Conclusion}

MDSCs can exert immunosuppressive effects under a variety of pathological conditions. In the field of organ transplantation, it is clinically important to understand that MDSCs play an immunosuppressive role and induce immune tolerance mechanisms. How to use MDSCs to induce the body to produce immune tolerance, thereby reducing the use of immunosuppressive agents and their toxic side effects, and prolonging the survival time of the graft will become the hot issues in the future clinical treatment strategies. 


\section{Acknowledgements}

This work was supported by Youth Project of National Natural Science Foundation (Grant No. 81200400) and National Social Science Foundation (Grant No. 14CFX031) of China. Top-notch innovative talents project of Fujian (Grant 2016Y9025\&2016J06018 \&2012J05148) Fujian Medical University teaching reform project (Y17005) and Fujian Provincial Health and Family planning Commission Youth Research Project (2017-1-6).

\section{Conflict of interest}

The authors have no financial conflicts of interest.

\section{References}

1. Youn JI, Gabrilovich DI. The biology of myeloid-derived suppressor cells: the blessing and the curse of morphological and functional heterogeneity. Eur J Immunol. 2010;40(11):2969-2975.

2. Mossadegh Keller N, Sarrazin S, Kandalla PK, et al. M-CSF instructs myeloid lineage fate in single haematopoietic stem cells. Nature. 2013;497(7448):239-243.

3. Alvarez Errico D, Vento Tormo R, Sieweke M, et al. Epigenetic control of myeloid cell differentiation, identity and function. Nat Rev Immunol. 2015;15(1):7-17.

4. Youn JI, Collazo M, Shalova IN, et al. Characterization of the nature of granulocytic myeloid-derived suppressor cells in tumor-bearing mice. $J$ Leukoc Biol. 2012;91(1):167-181.

5. Zhang H, Li Z, Wang L, et al. Critical Role of Myeloid-Derived Suppressor Cells in Tumor-Induced Liver Immune Suppression through Inhibition of NKT Cell Function. Front Immunol 2017;8:129.

6. Ochando JC, Chen SH. Myeloid-derived suppressor cells in transplantation and cancer. Immunol Res. 2012;54(3):275-285.

7. Guillonneau C. Efficacy of Myeloid Derived Suppressor Cells on Transplant Survival. Transplantation. 2015;99(10):2017-2019.

8. Lees JR, Azimzadeh AM, Bromberg JS. Myeloid derived suppressor cells in transplantation. Curr Opin in Immunol. 2011;23(5):692-697.

9. Gabrilovich DI, S Nagaraj. Myeloid-derived suppressor cells as regulators of the immune system. Nat Rev Immunol. 2009;9(3):162-174.

10. Ost M, Singh A, Peschel A, et al. Myeloid-Derived Suppressor Cells in Bacterial Infections. Front Cell Infect Microbiol. 2016;6:37.

11. Goh C, Narayanan S, Hahn YS. Myeloid-derived suppressor cells: the dark knight or the joker in viral infections? Immunol Rev. 2013;255:210-221.

12. Waight JD, Netherby C, Hensen ML, et al. Myeloid-derived suppressor cell development is regulated by a STAT/IRF-8 axis. J Clin Invest. 2013;123(10):4464-4478.

13. Dorhoi A, N Du Plessis. Monocytic Myeloid-Derived Suppressor Cells in Chronic Infections. Front Immunol. 2018;8:1895.

14. Zhou Z, French DL, Ma G, et al. Development and function of myeloid-derived suppressor cells generated from mouse embryonic and hematopoietic stem cells. Stem Cells. 2010;28(3):620-632.

15. Dilek N, Vuillefroy De Silly R, Blancho G, et al. Myeloid-derived suppressor cells: mechanisms of action and recent advances in their role in transplant tolerance. Front Immunol. 2012;3:208.

16. Wu T, Zhao $\mathrm{Y}$. The roles of myeloid-derived suppressor cells in transplantation. Expert Rev Clin Immunol. 2014;10(10):1385-1394.

17. Highfill SL, Rodriguez PC, Zhou Q, et al. Bone marrow myeloidderived suppressor cells (MDSCs) inhibit graft-versus-host disease (GVHD) via an arginase-1-dependent mechanism that is up-regulated by interleukin-13. Blood. 2010;116(25):5738-5747.
18. Rieber N, Wecker I, Neri D, et al. Extracorporeal photopheresis increases neutrophilic myeloid-derived suppressor cells in patients with GvHD. Bone Marrow Transplant. 2014;49(4):545-552.

19. Blancou P, Tardif V, Simon T, et al. Immunoregulatory Properties of Heme Oxygenase-1: Suppression and Regulation of Immune Responses. Methods in Molecular Biology. 2011;677:247-268.

20. Wang Y, Gu X, Xiang J, et al. Myeloid-derived suppressor cells participate in preventing graft rejection. Clin Dev Immunol. 2012;2012:731486.

21. Rosborough BR, Raïch Regué D, Turnquist HR, et al. Regulatory Myeloid Cells in Transplantation. Transplantation. 2014;97(4):367-379.

22. Hsieh CC, Chou HS, Yang HR, et al. The role of complement component 3 (C3) in differentiation of myeloid-derived suppressor cells. Blood. 2013;121(10):1760-1768.

23. Arakawa Y, Qin J, Chou HS, et al. Cotransplantation with myeloidderived suppressor cells protects cell transplants: a crucial role of inducible nitric oxide synthase. Transplantation. 2014;97(7):740-747.

24. Yi T, Li X, Yao S, et al. Host APCs Augment In Vivo Expansion of Donor Natural Regulatory T Cells via B7H1/B7.1 in Allogeneic recipients. $J$ Immunol. 2011;186(5):2739-2749.

25. Dilek N, Poirier N, Usal C, et al. Control of transplant tolerance and intragraft regulatory $\mathrm{T}$ cell localization by myeloid-derived suppressor cells and CCL5. J Immunol. 2012;188(9):4209-4216.

26. Garcia MR, Ledgerwood L, Yang Y, et al. Monocytic suppressive cells mediate cardiovascular transplantation tolerance in mice. J Clin Invest. 2010;120(7):2486-2496.

27. Chou HS, Hsieh CC, Charles R, et al. Myeloid-derived suppressor cells protect islet transplants by B7-H1 mediated enhancement of T regulatory cells. Transplantation. 2012;93(3):272-282.

28. Luan Y, Mosheir E, Menon MC, et al. Monocytic myeloidderived suppressor cells accumulate in renal transplant patients and mediate CD4(+) Foxp3(+) Treg expansion. Am J Transplant. 2013;13(12):3123-3131.

29. Park MJ, Lee SH, Kim EK, et al. Myeloid-Derived Suppressor Cells Induce the Expansion of Regulatory B Cells and Ameliorate Autoimmunity in the Sanroque Mouse Model of Systemic Lupus Erythematosus. Arthritis Rheumatol. 2016;68(11):2717-2727.

30. He Y, Bei J, Zeng H,. The roles of sepsis-induced myeloid derived suppressor cells in mice corneal, skin and combined transplantation. Transpl Immunol. 2016;34:8-13.

31. He Y, Wang B, Jia B, et al. Effects of Adoptive Transferring Different Sources of Myeloid-Derived Suppressor Cells in Mice Corneal Transplant Survival. Transplantation. 2015;99(10):2102-2108.

32. Marigo I, Bosio E, Solito S, et al. Tumor-induced tolerance and immune suppression depend on the $\mathrm{C} / \mathrm{EBPbeta}$ transcription factor. Immunity. 2010;32(6):790-802.

33. Qin J, Arakawa Y, Morita M, et al. C-C Chemokine Receptor type 2 (CCR2)-Dependent Migration of Myeloid-Derived Suppressor Cells in Protection of Islet Transplants. Transplantation. 2017;101(8):1793-1800.

34. Wu T, Zhao Y, Wang H, et al. mTOR masters monocytic myeloid-derived suppressor cells in mice with allografts or tumors. Sci Rep. 2016;6:20250.

35. Carretero Iglesia L, Bouchet Delbos L, Louvet C, et al. Comparative Study of the Immunoregulatory Capacity of In Vitro Generated Tolerogenic Dendritic Cells, Suppressor Macrophages, and MyeloidDerived Suppressor Cells. Transplantation. 2016;100:2079-2089.

36. Drujont L, Carretero Iglesia L, Bouchet Delbos L, et al. Evaluation of the therapeutic potential of bone marrow-derived myeloid suppressor cell (MDSC) adoptive transfer in mouse models of autoimmunity and allograft rejection. PLoS One. 2014;9(6):e100013. 